\title{
A CSOV Study of the Difference between HF and DFT Intermolecular Interaction Energy Values: The Importance of the Charge Transfer Contribution
}

\author{
JEAN-PHILIP PIQUEMAL, ${ }^{*}$ ANTONIO MARQUEZ, ${ }^{2}$ OLIVIER PARISEL,, 3 \\ CLAUDE GIESSNER-PRETTRE ${ }^{1}$ \\ ${ }^{1}$ Laboratoire de Chimie Théorique, UMR 7616-CNRS/UPMC, Université P. \& M. Curie, \\ Case courrier 137, 4 place Jussieu, F. 75252 Paris Cedex 05, France \\ ${ }^{2}$ Departamento de Quimica Fisica, Facultad de Quimica, Universitad de Sevilla, E-41012 \\ Sevilla, Spain \\ ${ }^{3}$ Laboratoire d'Etude Théorique des Milieux Extrêmes, Parc Valrose, Faculté des Sciences, \\ Université de Nice, F. 06108 Nice Cedex 2, France
}

Received 17 August 2004; Accepted 8 March 2005

DOI 10.1002/jcc.20242

Published online in Wiley InterScience (www.interscience.wiley.com).

\begin{abstract}
Intermolecular interaction energy decompositions using the Constrained Space Orbital Variation (CSOV) method are carried out at the Hartree-Fock level on the one hand and using DFT with usual GGA functionals on the other for a number of model complexes to analyze the role of electron correlation in the intermolecular stabilization energy. In addition to the overall stabilization, the results provide information on the variation, with respect to the computational level, of the different contributions to the interaction energy. The complexes studied are the water linear dimer, the $N$-methylformamide dimer, the nucleic acid base pairs, the benzene-methane and benzene- $\mathrm{N}_{2}$ van der Waals complexes, $\left[\mathrm{Cu}^{+}-(\operatorname{ImH})_{3}\right]_{2}$, where "ImH" stands for the Imidazole ligand, and ImH-Zn ${ }^{++}$. The variation of the frozen core energy (the sum of the intermolecular electrostatic energy and the Pauli repulsion energy) calculated from the unperturbed orbitals of the interacting entities indicates that the intramolecular correlation contributions can be stabilizing as well as destabilizing, and that general trends can be derived from the results obtained using usual density functionals. The most important difference between the values obtained from HF and DFT computations concerns the charge transfer contribution, which, in most cases, undergoes the largest increase. The physical meaning of these results is discussed. The present work gives reference calculations that might be used to parametrize new correlated molecular mechanics potentials.
\end{abstract}

(C) 2005 Wiley Periodicals, Inc. J Comput Chem 26: 1052-1062, 2005

Key words: intermolecular interaction energy decomposition; DFT vs. HF results; CSOV

\section{Introduction}

It is firmly established that electron correlation can be of primary importance for the stability of intermolecular complexes. When the contribution of electron correlation to the intermolecular interaction energy is calculated within the supermolecular approach, one does not get any information on the forces responsible for the variation of calculated energies going from a computational procedure to another, nor on the relative importance of the intra- and intermolecular contributions. ${ }^{1}$ The development of highly accurate intermolecular potentials requires, however, the knowledge of such quantities. Two main approaches are available for the decomposition of the interaction energy of weakly bound systems: the first one relies on perturbation treatments, and the second one is purely variational. Both construct the interaction energy by calculating different terms from the wave function of the separate monomers.

The reference method, the Symmetry-Adapted Perturbation Theory $\left(\mathrm{SAPT}^{2}\right)$, belongs to the first group and is able to give

\footnotetext{
Correspondence to: O. Parisel, e-mail: parisel@1ct.jussieu.fr

*Present address: Laboratory of Structural Biology, National Institute of Environmental Health Science, Mail Drop F0-08, P.O. Box 12233, Research Triangle Park, North Carolina 27709.

This article includes Supplementary Material available from the authors upon request or via the Internet at http:/www.interscience.wiley.com/ jpages/0192-8651/suppmat
} 
precise data about the intra- and intermolecular contributions to the intermolecular correlation energy. Here, the interaction energy is built in terms of perturbation orders (up to the third order $^{2 b}$ ). From such computations on various complexes, it appears that intramolecular and intermolecular contributions to correlation are of opposite sign: ${ }^{3,6-9}$ the overall stabilization obtained in most cases is due to the larger magnitude of the intermolecular terms. Unfortunately, their computational cost is prohibitive, and precludes using SAPT for force-field parametrization even if some attempts to simplify the problem has been considered within the Localized MP2 (LMP2) ${ }^{3-5}$ formalism or Stone's intermolecular perturbation theory (IMPT) ${ }^{1 \mathrm{~b}}$ decomposition. Nevertheless, these methods remain less accurate than SAPT and still require heavy time-consuming calculations.

On the other hand, it is possible to decompose the intermolecular interaction energy using variational methods such as HartreeFock (HF) or the Density Functional Theory (DFT). First developed by Morokuma ${ }^{10 a, b}$ and Ziegler, ${ }^{11}$ such methods decompose the interaction energy into physically meaningful contributions. ${ }^{10-12,13 a, 14-15}$ They are notoriously faster than the perturbation approaches, and can be seen as good approximations of these later ones. For example, $\mathrm{CSOV}^{12 \mathrm{a}, \mathrm{b}}$ and RVS (Restricted Variational Space ${ }^{13 a}$ ) build the molecular Fock operator of the supermolecule, or its Kohn-Sham (KS) equivalent, from the occupied and unoccupied orbitals of the interacting entities. These slightly different decomposition schemes all delete some integrals and elements of the Fock matrix: the corresponding SCF procedures provide the energies of interest. All approaches build a variational space upon the orbitals of the isolated monomers. This way it is possible to run constrained SCF procedures keeping some orbitals frozen. Then, according to the set of orbitals frozen, one gets the various contributions to the interaction energy.

Therefore, for a given complex, a CSOV energy decomposition can be carried out either at the HF or DFT level, ${ }^{12}$ the differences between the two sets of values can be attributed to the contribution of correlation to each term involved. However, it should be kept in mind that the DFT formalism does not give access to the dispersion terms. Nevertheless, there is accumulation of numerical examples showing that DFT calculations can give accurate results for nonpurely dispersion interactions, which are certainly the most frequent in chemistry. ${ }^{16}$ Such CSOV calculations should thus be able to give information on the correlation contribution to each of these terms provided that the HF and DFT computations are carried out using the same basis set.

The parameterization of some molecular mechanics systematics such as SIBFA (Sum of Interaction Between Fragment Ab Initio) ${ }^{17,18}$ relies on such energy decompositions performed at the HF level. The same procedure relying on DFT computations could provide a simple way to obtain parameters taking intra- and intermolecular correlation into account because the electrostatic and polarization terms could be calculated using "correlated" distributed multipoles ${ }^{19}$ and polarizabilities (J.-P. Piquemal, in preparation) using the same functional and basis set. We then thought that it could be of interest to carry out a series of energy decompositions at the HF and DFT levels for some intermolecular complexes using different GGA functionals and basis sets. The general trends of the results should give indications on the contribution of correlation to the interaction energy.
In this study we will consider different types of complexes. The first set is representative of interactions through hydrogen bonding and includes the linear water dimer, which was used in pioneering calculations of charge-transfer, ${ }^{10 f}$ the $N$-methylformamide dimer, and the nucleic acid base pairs. The second group includes van der Waals complexes such as benzene-methane and benzene- $\mathrm{N}_{2}$, for which the PW91 functional has been shown to give reliable numerical results. ${ }^{20}$ The computations are extended to two complexes involving metals. The first one is a system that gives rather unexpected results at the DFT and MP2 levels when compared to those obtained from $\mathrm{HF}$ computations: two interacting $\mathrm{Cu}^{+}-$ $(\mathrm{ImH})_{3}$ entities, where "ImH" stands for the imidazole ligand: despite the presence of a net positive charge on each of the two interacting units, a metastable arrangement has been found when correlation is taken into account. ${ }^{21,22}$ The second one is the $\mathrm{Zn}^{++}$ ImH complex.

\section{Computational Details}

The intermolecular interaction energy decompositions are carried out within the $\mathrm{CSOV}^{12}$ method. This procedure is affiliated to Morokuma's: ${ }^{10 a, b}$ it calculates the same components of the interaction energy but differs in the construction of the Fock matrices to be used for the computation of the polarization and charge transfer terms. Morokuma's scheme, starting from the molecular orbitals of the isolated fragments (occupied and virtual), suppresses successively selected blocks of the complete Fock matrix and performs different SCF procedures giving the various components of the intermolecular interaction energy. The CSOV procedure for the construction of the different Fock matrices, or their KS equivalent, is slightly different when dealing with the polarization and charge transfer components. Using McWeeny's group function approach, ${ }^{13 \mathrm{~b}-\mathrm{d}}$ which constructs an A-B complex upon the orbitals of the isolated monomers A and $\mathrm{B}$, it is possible to select variational spaces including or excluding the virtual orbitals of $\mathrm{A}$ or $\mathrm{B}$ in the construction of A-B Fock matrix or to freeze the orbitals of a chosen monomer. From this computational procedure one gets two types of contributions depending on the choice of the variational space. The "frozen core" energy $\left(E_{\mathrm{FC}}\right)$, which is the sum of the electrostatic $\left(E_{s}\right)$ and exchange/Pauli repulsion $\left(E_{\text {Pauli }}\right)$ terms, is calculated, as in Morokuma's scheme, from the molecular orbitals of the isolated interacting entities, and corresponds to the antisymmetrized Hartree product of the isolated monomer wave functions, $\hat{A}\left(\Psi_{0}^{\mathrm{A}} \Psi_{0}^{\mathrm{B}}\right)$ where $\hat{A}$ is the antisymmetrisation operator. In that case, the occupied orbitals of $A$ and $B$ remain frozen with no possibility of relaxation into the virtual orbitals which are excluded from the SCF procedure. At the HF level, the CSOV values for $E_{s}$ and $E_{\text {Pauli }}$ are identical to Morokuma's $E_{s}$ and $E_{\mathrm{xr}}$.

The electrostatic energy corresponds to the classic Coulomb interaction of the electron distributions of the isolated monomers, kept "frozen," that is, not perturbed, into the A-B complex arrangement. $E_{s}$ includes a short range term, ${ }^{18 \mathrm{a}, \mathrm{b}}$ related to the penetration of the charge distributions, and a long-range multipolar component, ${ }^{1 \mathrm{a}, 17,18 \mathrm{a}, \mathrm{b}}$ which is usually approximated by a multipolar expansion. In the CSOV approach, the computation of $E_{s}$ requires only the evaluation of the three following quantities: the electron-electron repulsion, the electron-nucleus attraction, and the nucleus-nucleus repulsion energy, ${ }^{18 \mathrm{a}-\mathrm{c}}$ 


$$
\begin{aligned}
\left.E_{s}=-2 \sum_{i} \sum_{v} Z_{v} \int\left(\left|\varphi_{i}(1)\right|^{2}\right) / r_{1 \nu}\right) d \mathrm{l}_{1} \\
\\
\left.\quad-2 \sum_{j} \sum_{\mu} Z_{\mu} \int\left(\left|\varphi_{j}(2)\right|^{2}\right) / r_{2 \mu}\right) d \mathrm{l}_{2} \\
\left.+4 \sum_{i} \sum_{j} \int\left(\left|\varphi_{i}(1)\right|^{2}\left|\varphi_{j}(2)\right|^{2}\right) / r_{12}\right) d \mathrm{l}_{1} d \mathrm{l}_{2}+\sum_{\mu} \sum_{v} Z_{\mu} \cdot Z_{v} / r_{\mu \nu}
\end{aligned}
$$

where $\mu$ and $\varphi_{i}$ are, respectively, the nucleus and the unperturbed molecular orbitals of monomer $\mathrm{A}$ and $\nu$, and $\varphi_{j}$ are the equivalents for monomer B.

The exchange-repulsion term, which results from Pauli exclusion principle, is calculated as the difference between $E_{\mathrm{FC}}$ and $E_{s}$. Therefore, the differences between the values obtained from $\mathrm{HF}$ and DFT computations, for these two contributions, can be attributed to intramolecular correlation, as "handled" by DFT. The other terms, "nonfrozen terms," are the polarization $\left(E_{\mathrm{pol}}\right)$ and charge transfer $\left(E_{\mathrm{ct}}\right)$ contributions, which depend upon the variation of the molecular orbital and their eigenvalues due to the intermolecular interaction. For a molecule A interacting with a molecule B, $E_{\text {polA }}$ corresponds to the relaxation of the occupied orbitals of molecule A by the electric field generated by molecule B. Molecule B occupied orbitals remain frozen and its virtual orbitals are excluded from the SCF procedure performed on molecule A. Molecule A orbitals are kept orthonormal to the frozen MO's of B.

The charge-transfer contribution, $E_{\mathrm{ctA} \rightarrow \mathrm{B}}$, is calculated by a similar procedure but this time the virtual orbitals of $B$ are included into the variational space, allowing the possibility of charge transfer. Here, it is important to point out that both polarization and charge-transfer contributions are computed using antisymmetrized wave functions to avoid Morokuma decomposition short-range problem in complexes involving strong polarizing field. ${ }^{10 e}$

Finally, at both computational levels (HF and DFT) we have:

$$
\begin{aligned}
E_{\mathrm{tot}}=E_{\mathrm{FC}}+E_{\mathrm{pol}}+E_{\mathrm{ct}}=E_{s}+E_{\mathrm{Pauli}}+ & E_{\mathrm{polA}} \\
& +E_{\mathrm{polB}}+E_{\mathrm{ctA} \rightarrow \mathrm{B}}+E_{\mathrm{ctB} \rightarrow \mathrm{A}}
\end{aligned}
$$

At this point we should point out that $E_{\text {tot }}$ differs from the intermolecular interaction energy value, $\Delta E$, calculated from the difference between the supermolecule energy and the sum of the energies of the interacting entities. The missing terms (thereafter called $\delta E$ ), which are not included in the CSOV treatment have different physical origins. Some are related to the needed antisymmetrization of the wave function, and are specific exchange terms that have been extensively discussed in the SAPT theory ${ }^{10 \mathrm{e}}$ and in various articles. ${ }^{10 a, b, 13 a}$ Others are related to the fact that, for the polarization and charge-transfer energies, the procedure neglects the induced dipoles created on A and B. Usually, these many-body terms remain very small and are not determined by the "standard" CSOV analysis. Because of the differences in the calculation of $E_{\mathrm{pol}}$ and $E_{\mathrm{ct}}$ between the Morokuma and CSOV schemes, the $\delta E$ term related to the two methods are different (see ref. 10e for a very complete presentation).

In addition to the results of the CSOV computations, we also report for most of the systems studied the value of the Basis Set
Superposition Error (BSSE) to have an estimation of the quality of the basis set used, and therefore of the interaction energy value. These results are important to the discussion because the BSSE appears to be located in the charge-transfer component of the interaction energy. ${ }^{1 \mathrm{a}, \mathrm{b}, 13 \mathrm{a}}$

$$
\begin{gathered}
E_{\mathrm{tot}(\mathrm{BSSE} \text { corrected })}=E_{\mathrm{FC}}+E_{\mathrm{pol}}+\left(E_{\mathrm{ct}}-\mathrm{BSSE}\right) \\
\Delta E_{(\mathrm{BSSE} \text { corrected })}=E_{\mathrm{tot}(\mathrm{BSSE} \text { corrected })}+\delta E^{\prime}
\end{gathered}
$$

The interaction energy values calculated using the $\operatorname{CCSD}(\mathrm{T})$ method chosen as the reference (or at the MP2 level because of the computational cost) will allow to estimate the error due to the use of DFT because this method has been shown to tend to underestimate, for systems such as van der Waals complexes, ${ }^{23}$ the correlation contribution to intermolecular interaction energies.

The CSOV computations are carried out using a modified version of HONDO95.3. ${ }^{24}$ The CCSD(T), MP2 (full, 6d) computations and the BSSE corrections as well as the molecular polarizabilities are calculated using GAUSSIAN03. ${ }^{25}$ The GGA functionals retained for this study are BLYP and PW91 (PW91PW91) on the one hand and the corresponding hybrid B3LYP and B3PW91 on the other. The different basis sets are: aug-cc-pVTZ, ${ }^{26}$ $6-31++\mathrm{G}(\mathrm{d}, \mathrm{p}),{ }^{27,28} 6-311++\mathrm{G}(\mathrm{d}, \mathrm{p}),{ }^{28,29}$ and DZVP2 ${ }^{30}$ basis sets. The first one was retained because it has been shown to give reliable DFT intermolecular interaction energy values and minimum BSSE corrections. ${ }^{16}$ The choice of the $6-311++\mathrm{G}(\mathrm{d}, \mathrm{p})$ and $6-31++\mathrm{G}(\mathrm{d}, \mathrm{p})$ basis sets relies on their wide use for a large variety of systems. The DZVP2 basis set, optimized from DFT computations, could provide, from comparison with the results given by the $6-31++\mathrm{G}(\mathrm{d}, \mathrm{p})$ basis, indications on the influence of the optimization framework because both are split polarized bases. For the largest complex studied only the DZVP2 basis set is used and the "correlated" energy decomposition is limited to B3LYP because of computational cost.

The geometrical arrangements considered are shown on Figure 1 . For the water dimer the intramolecular geometry of the monomers is that of the isolated molecule $\left(0.96 \AA\right.$ and $\left.104.5^{\circ}\right)$, the $\mathrm{O}-\mathrm{O}$ distance is equal to $2.96 \AA$, and the tilt angle of molecule A with respect to proton donating $\mathrm{OH}$ bond of molecule B is $50.0^{\circ}$. The intermolecular distance in the $N$-methylformamide dimer is equal to $2.00 \AA$. The geometry used for the nucleic acid base pairs adenine-thymine (AT) and guaninecytosine (GC) are taken from a previous study. ${ }^{31}$ For the benzene- $\mathrm{N}_{2}$ complex the geometry is that optimized by Wesolowski et al. ${ }^{20}$ for the same arrangement using the PW91 functional: it has an intermolecular distance of $3.56 \AA$. For $\left[\mathrm{Cu}^{+}-(\mathrm{ImH})_{3}\right]_{2}$ we retained the bond lengths and angles used in ref. 21. In the ImH- $\mathrm{Zn}^{++}$complex the $\mathrm{Zn}-\mathrm{N}$ distance is equal to $1.90 \AA$. All geometries are given in the Supplementary Material Section as sets of Cartesian coordinates.

\section{Results and Discussion}

\section{The Linear Water Dimer}

The results concerning the linear water dimer are reported in Table 1. They show that, as expected, the interaction energy values 


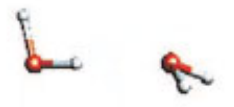

a
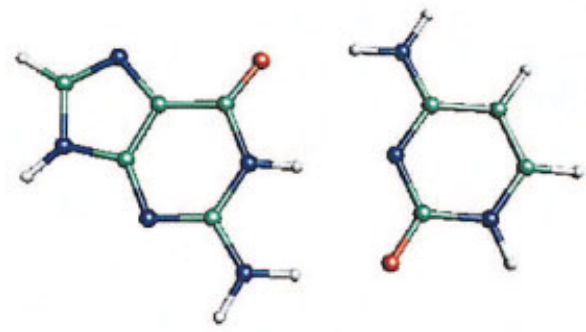

c

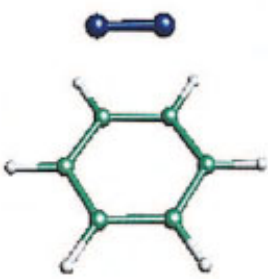

f
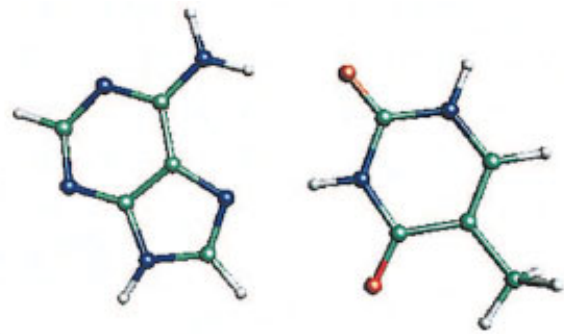

b

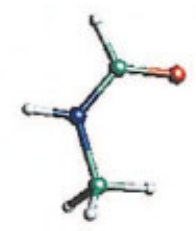

$\mathbf{e}$

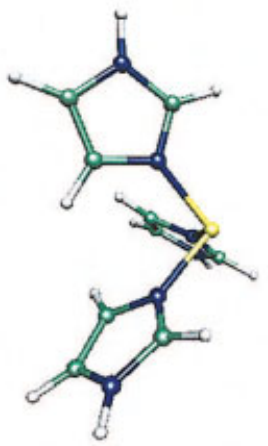

d
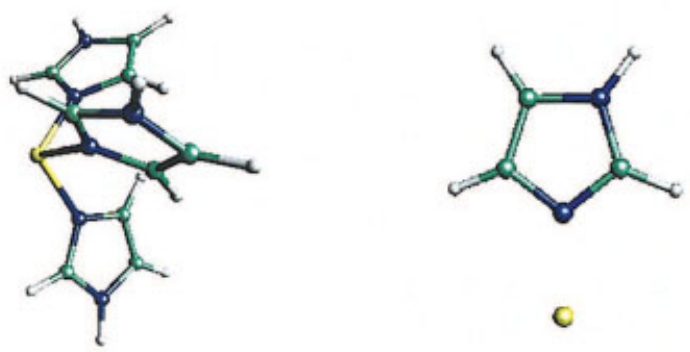

0

Figure 1. Geometrical arrangement of the different complexes considered: (a) water dimer, (b) adenine-thymine base pair, (c) guanine-cytosine base pair, (d) $\mathrm{N}$-methylformamide dimer, (e) benzene- $\mathrm{CH}_{4}$, (f) benzene- $\mathrm{N}_{2},(\mathrm{~g})\left[\mathrm{Cu}^{+}-(\mathrm{ImH})_{3}\right]_{2}$, (h) imidazole- $\mathrm{Zn}^{++}$.

calculated using DFT are larger than those obtained at the HF level and are in good agreement with those obtained at the $\operatorname{CCSD}(\mathrm{T})$ level of calculation, especially if using the PW91 functional.

With all the basis sets considered the largest increase of the intermolecular interaction energy occurs for the PW91 functional, as obtained previously ${ }^{16,20}$ for other complexes, followed by the B3LYP hybrid one. The most striking difference between the HF and DFT CSOV results concerns the charge transfer contribution from the proton acceptor to the proton donor, which is considerably increased when correlation is taken into account. A similar trend has been observed, for example, in the cases of $\mathrm{CO}$ or $\mathrm{NH}_{3}$ interacting with copper. ${ }^{12 \mathrm{e}}$ In our case, with the basis sets retained, this term is multiplied by a factor which can rise up to 2 . It is important to note that this increase cannot be associated with a BSSE artifact: for the most extended basis set, aug-cc-PVTZ, where the BSSE is close to zero, this increase is still obtained. The 


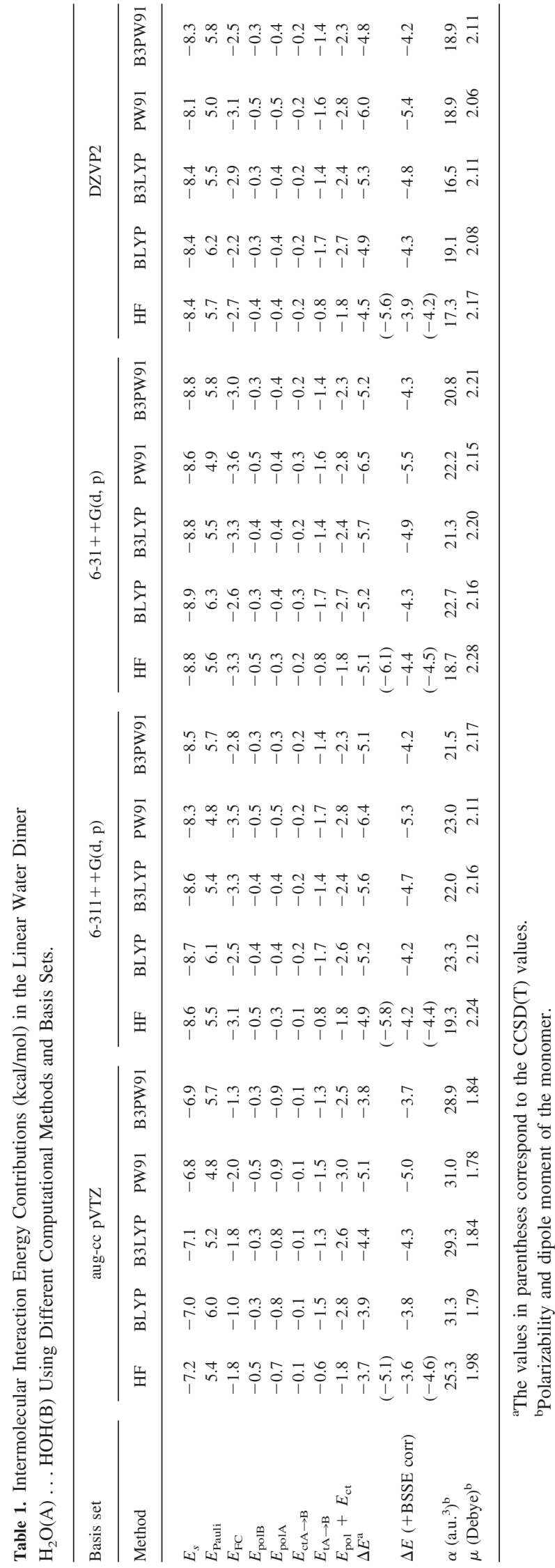

results show that the molecular dipole moment is decreased with respect to the HF value when calculated from DFT. On the contrary, the molecular polarizability increases when going from HF to DFT (see ref. 16 for extensive bibliography). Because of the opposite variations of the polarizability and dipole moment, ${ }^{16}$ the polarization contribution to the interaction energy increases or decreases depending on the functional used. The values of Table 1 also show that the DZVP2 basis gives results quite similar to those obtained with the $6-311++\mathrm{G}(\mathrm{d}, \mathrm{p})$ and $6-31++\mathrm{G}(\mathrm{d}, \mathrm{p})$ basis, a feature that illustrates that the optimization framework of the bases set has only a minor influence on the final energy components. Moreover, the inclusion of the BSSE corrections usually makes the DFT and the CCSD(T) calculations in good agreement. For PW91, however, the consideration of BSSE slightly diminish the agreement with the $\operatorname{CCSD}(\mathrm{T})$ results.

For this water dimer our results clearly indicate that the greater increase of stabilization (negative value of the energy) due to the correlation recovered at the DFT level appears in the polarization and charge transfer energies that are the intermolecular part of the correlation contribution to the intermolecular interaction energy. Moreover, strong correlation effects can be observed for the frozen core energy $\left(E_{\mathrm{FC}}\right)$ and the tabulated values show the importance of "frozen" terms, which depend on the initial charge distributions. The rules governing the variation of $E_{\mathrm{FC}}$, which corresponds to the intramolecular correlation contribution, are complex. It appears necessary to have a deeper insight into the changes in the electrostatic and Pauli repulsion components of the energy.

For $E_{s}$, the general trend of the DFT results (the exceptions will be discussed) shows a decrease of the stabilization when adding correlation. This is understandable, since the HF level generally overestimates this quantity because of its well-known overestimation of the dipole component of the charge distribution. ${ }^{12 \mathrm{e}}$ All functionals perform better than $\mathrm{HF}$ in reproducing the dipole moment of the monomer (the experimental value is 1.85 Debye), the best agreement is obtained for the B3LYP functional associated with the aug-cc-pVTZ basis set. For this extended basis set all DFT results show a decrease of the $E_{s}$ energy in agreement with the more sophisticated SAPT results. This stresses the importance of adding diffuse functions to the basis set to reproduce such a property. Nevertheless, for smaller basis set, the functionals do not perform equally. The largest change occurs for the PW91 functional. The hybrid functionals (B3LYP and B3PW91) and PW91 always follow the general trend giving equal or smaller values of $E_{s}$ than at the HF level; it is not the case of BLYP. Indeed, this functional gives greater electrostatic energies for both $6-31++\mathrm{G}(d, p)$ and $6-311++\mathrm{G}(d, p)$ basis sets. Surprisingly, the dipole moment values calculated with these bases are in fair agreement with those given by the other functionals. At this point, it is also important to stress upon the behavior of the PW91 functional for electrostatic interactions: it gives the lowest $E_{s}$ absolute value with all the basis sets used. For the exchange energy, PW91 leads to the smallest values whatever the basis set and, like that for $E_{s}$, presents the greatest variation when compared to HF values. It is closely followed by the BLYP functional but with an opposite behavior: the BLYP $E_{\text {Pauli }}$ energies are always more repulsive than those obtained at the HF level. The changes with the hybrid functionals are more moderate, but differ upon the correlation functional: surprisingly, B3LYP follows the trend of PW91 and B3PW91 that of BLYP.

The overall results show that the BLYP and B3PW91 functionals lead to a less stabilizing $E_{\mathrm{FC}}$ than $\mathrm{HF}$ and that B3LYP and 
Table 2. DNA Base Pairs Interaction Energy Decomposition (kcal/mol) Using DZVP2 Basis Set.

\begin{tabular}{|c|c|c|c|c|c|c|c|c|c|c|}
\hline \multirow{2}{*}{$\begin{array}{l}\text { Complex } \\
\text { Method }\end{array}$} & \multicolumn{5}{|c|}{ Adenine(A) ... Thymine(B) } & \multicolumn{5}{|c|}{ Guanine(A) ... Cytosine(B) } \\
\hline & HF (MP2) & B3LYP & BLYP & B3PW91 & PW91 & HF (MP2) & B3LYP & BLYP & B3PW91 & PW91 \\
\hline$E_{s}$ & -21.3 & -20.1 & -19.9 & -20.0 & -19.3 & -35.6 & -32.8 & -32.3 & -32.6 & -31.4 \\
\hline$E_{\text {Pauli }}$ & 16.6 & 15.5 & 17.3 & 16.1 & 14.3 & 20.1 & 19.4 & 20.8 & 19.9 & 18.3 \\
\hline$E_{\mathrm{FC}}$ & -4.8 & -4.6 & -2.6 & -3.8 & -5.0 & -15.5 & -13.4 & -11.4 & -12.7 & -13.1 \\
\hline$E_{\mathrm{polB}}$ & -1.2 & -1.2 & -1.2 & -1.0 & -1.4 & -3.3 & -3.3 & -3.3 & -3.3 & -3.5 \\
\hline$E_{\text {polA }}$ & -1.2 & -1.8 & -1.7 & -1.7 & -1.9 & -3.1 & -3.8 & -3.9 & -3.5 & -4.1 \\
\hline$E_{\mathrm{ctB} \rightarrow \mathrm{A}}$ & -1.6 & -1.7 & -2.2 & -1.8 & -2.1 & -4.0 & -5.0 & -5.9 & -4.9 & -5.7 \\
\hline$E_{\mathrm{ctA} \rightarrow \mathrm{B}}$ & -2.9 & -4.6 & -5.4 & -4.5 & -5.1 & -1.6 & -2.7 & -3.3 & -2.6 & -3.1 \\
\hline$E_{\mathrm{pol}}+\mathrm{E}_{\mathrm{ct}}$ & -7.0 & -9.3 & -10.4 & -8.9 & -10.4 & -12.0 & -14.9 & -16.4 & -14.3 & -16.4 \\
\hline$\Delta E^{\mathrm{a}}$ & $\begin{array}{c}-11.8 \\
(-19.1)\end{array}$ & -14.1 & -13.3 & -12.8 & -15.7 & $\begin{array}{c}-27.7 \\
(-33.6)\end{array}$ & -28.5 & -28.1 & -27.2 & -29.8 \\
\hline $\operatorname{BSSE}^{\mathrm{a}}$ & $\begin{array}{c}1.54 \\
(5.9)\end{array}$ & 1.63 & 1.93 & 1.44 & 1.73 & $\begin{array}{r}1.97 \\
(7.1)\end{array}$ & 2.17 & 2.25 & 1.84 & 2.09 \\
\hline$\alpha_{\mathrm{A}}\left(\mathrm{au}^{3}\right)$ & 79.8 & 85.2 & 87.31 & 85.06 & 87.0 & 85.6 & 93.4 & 96.48 & 93.07 & 96.03 \\
\hline$\alpha_{\mathrm{B}}\left(\mathrm{au}^{3}\right)$ & 69.6 & 75.3 & 77.76 & 77.4 & 74.92 & 64.6 & 69.7 & 71.78 & 69.49 & 71.43 \\
\hline$\mu_{\mathrm{A}}(\mathrm{D})$ & 2.59 & 2.48 & 2.45 & 2.50 & 2.43 & 7.93 & 7.56 & 7.47 & 7.54 & 7.42 \\
\hline$\mu_{\mathrm{B}}(\mathrm{D})$ & 5.17 & 4.79 & 4.68 & 4.75 & 4.63 & 8.10 & 7.41 & 7.18 & 7.35 & 7.10 \\
\hline
\end{tabular}

${ }^{\text {a }}$ The values in parentheses correspond to the MP2 values.

PW91 are able to gain energy. When compared to the HF values, the loss of $E_{\mathrm{FC}}$ energy for BLYP and B3PW91 are partially overcome by the increase of the corresponding polarization and charge transfer energies; however, the final gain remains modest compared to the B3LYP and PW91 values that both gain energy at the $E_{\mathrm{FC}}$ and $E_{\mathrm{pol}}+E_{\mathrm{ct}}$ levels. The PW91 total energy value is intriguing and appears too large when compared to the smaller $\operatorname{CCSD}(\mathrm{T})$ interaction energies in the more extended basis set; the comparison is more difficult for small basis sets because the $\operatorname{CCSD}(\mathrm{T})$ needs a relatively extended basis set to recover an important part of the correlation energy. The Pauli repulsion seems to be responsible for such an overestimation of the complexation energy when using PW91. Recent SAPT results ${ }^{32}$ on a very similar geometry of the water dimer show that the SAPT equivalent of $E_{\mathrm{FC}}$ (see refs. 2c and 13e for comparison between SAPT and variational decomposition schemes) is close to the B3LYP value for a MP3 level of theory. These SAPT calculations also show that, for an higher level of correlation treatment (CCSD correction), the $E_{\mathrm{FC}}$ value becomes more repulsive, and arises from a strong intramolecular redistribution of the density, leading to larger exchange values in agreement with other studies. ${ }^{3,6,9}$ Clearly, here, the BLYP and B3PW91 functionals seem to have the correct behavior (which does not imply that they are correct), on the contrary to the B3LYP and PW91 functionals. For B3LYP, this trend is limited by the small Pauli repulsion changes compared to the HF; it is not the case for PW91. Even if some problems remain for a direct comparison between CSOV and SAPT, such as the choice of the basis set (SAPT calculations use complete basis set extrapolation schemes) or the different operators used, the comparison is valid only for extended basis ${ }^{2 \mathrm{c}}$ sets such as aug-ccpVTZ. For this reason, the PW91 value appears to be particularly far away from the expected value.

\section{The DNA Base Pairs and the N-Methylformamide Dimer}

We have also investigated other hydrogen bonded complexes such as the DNA base pairs (Table 2) and $N$-methylformamide dimer (Table 3). The general rules observed for the water dimer remain the same. A decrease of the $E_{s}$ energy, which is the largest stabilizing term, is observed when going from HF to DFT. This variation increases with the magnitude of the contribution. As for the water dimer, a very different behavior occurs for $E_{\text {Pauli }}$ : PW91 gives the same weak value when compared to HF; BLYP enhances it. For all calculations the charge transfer contribution is increased at the DFT level, and this increase, as for the water dimer, cannot

Table 3. $N$-Methylformamide Dimer Interaction Energy Decomposition ( $\mathrm{kcal} / \mathrm{mol}$ ) Using the DZVP2 Basis Set.

\begin{tabular}{lrrrrr}
\hline Method & HF (MP2) & BLYP & B3LYP & PW91 & B3PW91 \\
\hline$E_{s}$ & -10.5 & -9.7 & -9.8 & -9.3 & -9.7 \\
$E_{\text {Pauli }}$ & 6.3 & 6.8 & 6.0 & 5.4 & 6.3 \\
$E_{\mathrm{FC}}$ & -4.1 & -2.8 & -3.8 & -3.8 & -3.3 \\
$E_{\mathrm{polB}}$ & -0.9 & -0.7 & -0.7 & -0.8 & -0.6 \\
$E_{\mathrm{polA}}$ & -0.7 & -0.8 & -0.8 & -0.8 & -0.8 \\
$E_{\mathrm{ctB} \rightarrow \mathrm{A}}$ & -0.5 & -0.7 & -0.6 & -0.7 & -0.5 \\
$E_{\mathrm{ctA} \rightarrow \mathrm{B}}$ & -1.0 & -2.2 & -1.8 & -2.1 & -1.8 \\
$E_{\mathrm{pol}}+E_{\mathrm{ct}}$ & -3.1 & -4.4 & -3.9 & -4.4 & -3.7 \\
$\Delta E^{\mathrm{a}}$ & -7.3 & -7.3 & -7.8 & -8.4 & -7.1 \\
& $(-9.3)$ & & & & \\
$\mathrm{BSSE}^{\mathrm{a}}$ & 0.82 & 1.01 & 0.86 & 0.94 & 0.78 \\
$\alpha_{\mathrm{A}}\left(=\alpha_{\mathrm{B}}, \mathrm{au}^{3}\right)$ & $(2.6)$ & & & & \\
$\mu_{\mathrm{A}}\left(=\mu_{\mathrm{B}}, \mathrm{D}\right)$ & 36.9 & 34.92 & 36.6 & 34.76 & 33.55 \\
\hline
\end{tabular}

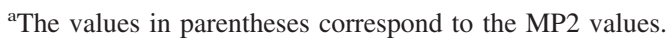


be related to BSSE differences between the two methods because it is almost the same at both the HF and DFT levels.

Our results for the base pairs are in qualitative agreement with those of intermolecular interaction energy decompositions ${ }^{33}$ carried out within the framework of the Amsterdam Density Functional (ADF) program. ${ }^{34}$ From both studies, the electrostatic term is larger than the total binding energy, and the DFT total interaction energies are of a comparable magnitude. However, there are important differences between the results obtained by Fonseca Guerra et al. ${ }^{33}$ and ours. With the BP86 functional and the TZ2P basis set the repulsion term from the ADF treatment is larger than the electrostatic term, leading to a repulsive $E_{\mathrm{FC}}$, while this sum is attractive from our calculations. The ADF numerical magnitude of the equivalent value of $E_{\mathrm{pol}}+E_{\mathrm{ct}}$ is more than twice larger than the corresponding values reported in Table 2. To specify these comments, it is useful to remember that the ADF treatment relies on the Ziegler decomposition scheme, which decomposes the energy as:

$$
E_{\text {int }}=\Delta V_{\text {elec }}^{0}+\Delta E_{\text {Pauli }}+\Delta E_{\mathrm{OI}}
$$

where $\Delta V_{\text {elec }}^{0}$ and $\Delta E_{\text {Pauli }}$ have exactly the same physical meaning as $E_{s}$ and $E_{\text {Pauli }}$ in the CSOV formalism. $\Delta E_{\mathrm{OI}}$ or "Orbital Interaction" correspond to the remaining energy, and is thus directly related to polarization and charge transfer CSOV energies. The differences are probably linked to the different functionals used in ADF that may present a different behavior for the Pauli repulsion, to the use of Slater atomic orbitals, which are considerably more extended than Gaussian basis sets, or, more probably, to differences in the geometries used. We wish to thank one of the referee who has checked this point by performing a number a complexation energy decompositions with ADF and the TZ2P+ basis set using the geometries reported here as Supplementary Materials. For the guanine-cytosine pair, the following decomposition is obtained with the BP86 functional (kcal/mol) $E_{s}=-30.7, E_{\text {Pauli }}$ $=21.3, E_{\mathrm{pol}}+E_{\mathrm{ct}}=-16.2$. With the PW91 and BLYP functionals, these components are respectively: $-30.9,19.2$, -16.1 , and $-30.5,18.5,-15.9$. For the adenine-thymine base pair, the results are as follows: $-19.9,15.6$, and -9.9 for PW91;

Table 4. Benzene(A)-Methane(B) Complex Interaction Energy Decomposition ( $\mathrm{kcal} / \mathrm{mol}$ ) Using the DZVP2 Basis Set.

\begin{tabular}{lcrrrr}
\hline Method & HF (MP2) & BLYP & B3LYP & PW91 & B3PW91 \\
\hline$E_{s}$ & -0.4 & -0.4 & -0.4 & -0.2 & -0.2 \\
$E_{\mathrm{Pauli}}$ & 1.3 & 1.5 & 1.2 & 0.2 & 1.4 \\
$E_{\mathrm{FC}}$ & 0.9 & 1.1 & 0.8 & 0.0 & 1.2 \\
$E_{\mathrm{polB}}$ & 0.0 & 0.0 & 0.0 & 0.0 & 0.0 \\
$E_{\mathrm{polA}}$ & 0.0 & 0.0 & 0.0 & -0.1 & 0.0 \\
$E_{\mathrm{ctB} \rightarrow \mathrm{A}}$ & 0.0 & 0.0 & 0.0 & 0.0 & 0.0 \\
$E_{\mathrm{ctA} \rightarrow \mathrm{B}}$ & -0.2 & -0.1 & -0.1 & -0.1 & -0.1 \\
$E_{\mathrm{pol}}+E_{\mathrm{ct}}$ & -0.2 & -0.1 & -0.1 & -0.3 & -0.1 \\
$\Delta E^{\mathrm{a}}$ & 0.6 & 1.0 & 0.7 & -0.3 & 1.0 \\
& $(-0.7)$ & & & & \\
$\Delta E+\mathrm{BSSE}^{\mathrm{a}}$ & 0.8 & 1.2 & 0.8 & -0.2 & 1.1 \\
& $(0.7)$ & & & & \\
\hline
\end{tabular}

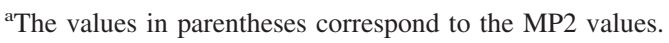

Table 5. Benzene- $\mathrm{N}_{2}$ Complex: Interaction Energy Decomposition (kcal/ mol) Using the aug-cc-pVTZ Basis (No f Orbitals).

\begin{tabular}{lcrrrr}
\hline Method & HF (MP2) & BLYP & B3LYP & PW91 & B3PW91 \\
\hline$E_{s}$ & -0.64 & -0.68 & -0.65 & -0.62 & -0.60 \\
$E_{\mathrm{Pauli}}$ & 1.46 & 5.02 & 1.41 & 0.47 & 1.69 \\
$E_{\mathrm{FC}}$ & 0.82 & 4.3 & 0.76 & -0.15 & 1.09 \\
$E_{\mathrm{polBenz}}$ & -0.03 & -0.03 & -0.03 & -0.05 & -0.03 \\
$E_{\mathrm{polN} 2}$ & -0.05 & -0.06 & -0.05 & -0.09 & -0.06 \\
$E_{\mathrm{ctBenz} \rightarrow \mathrm{N} 2}$ & -0.16 & -0.32 & -0.26 & -0.28 & -0.24 \\
$E_{\mathrm{ctN} 2 \rightarrow \mathrm{Benz}}$ & -0.07 & -0.10 & -0.09 & -0.10 & -0.10 \\
$E_{\mathrm{pol}}+E_{\mathrm{ct}}$ & -0.31 & -0.51 & -0.43 & -0.51 & -0.41 \\
$\Delta E^{\mathrm{a}}$ & 0.51 & 3.81 & 0.33 & -0.67 & 0.67 \\
& $(-1.95)$ & & & & \\
$\Delta E+\mathrm{BSSE}^{\mathrm{a}}$ & 0.52 & 3.91 & 0.44 & -0.56 & 0.11 \\
& $(-0.94)$ & & & & \\
\hline
\end{tabular}

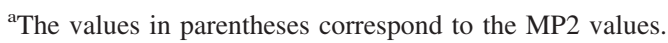

$-19.4,15.3$, and -9.8 for BLYP. These numbers are in very good agreement with the present CSOV decompositions. They seem to indicate that differences in the geometries used are the main reason for the discrepancy between Fonseca Guerra's results and ours.

\section{The Benzene-Methane Complex}

The benzene-methane complex (see Table 4) gives interesting results because this system lacks polarization due to two apolar fragments. Indeed, this kind of complex, as well as the benzene- $\mathrm{N}_{2}$ complex which will be discussed later, is also important because of the lack of purely dispersive terms in standard DFT GGA calculations. As expected, the polarization energies are null: only the PW91 functional finds a nonzero polarization contribution $(-0.1$ $\mathrm{kcal} / \mathrm{mol}$ ). The charge transfer contribution is also weak and is exactly of the same magnitude as the BSSE: it might not be relevant because it is highly contaminated. For all functionals, $E_{s}$ is equally or less attractive than at the HF level, as discussed previously. The Pauli repulsion shows large differences between the different functionals, even if the rules remain the same as in the hydrogen bonded systems. Hybrid functional results are close to the HF value, the BLYP and PW91 functionals give again very opposite results. BLYP shows a more repulsive $E_{\text {Pauli }}$ than $\mathrm{HF}$ whereas PW91 leads to a very weak $E_{\text {Pauli }}$ energy $(0.2 \mathrm{kcal} / \mathrm{mol})$. This weak $E_{\text {Pauli }}$ makes PW91 predict a bound complex $(\Delta E=$ $-0.3 \mathrm{kcal} / \mathrm{mol}$, which becomes -0.2 when BSSE is taken into account), a somewhat unphysical result because the post-HF MP2 and the other functionals give a repulsive interaction when BSSE is taken into account. It can be seen that PW91 is the only functional leading to nonzero polarization energy for the benzene. The opposite trend leads BLYP to give a too repulsive energy, and only the hybrid functionals are in reasonable agreement with the MP2 values.

\section{The Benzene- $\mathrm{N}_{2}$ Complex}

For the benzene- $\mathrm{N}_{2}$ complex (see Table 5), the computations were carried out using the aug-cc-pVTZ basis set, omitting the f orbitals 
because of computational limitations. The choice of this basis set is guided by its ability to decrease the BSSE error. Such a large basis is required for complexes characterized by small interaction energies. All DFT calculations but PW91 fail to reproduce the MP2 interaction energy. The results reported in Table 5 show that the PW91 functional is the only one leading to a stable complex. If the BSSE correction is taken into account, the MP2 interaction energy falls within $0.5 \mathrm{kcal} / \mathrm{mol}$ from the corrected PW91 value. $E_{\mathrm{FC}}$, which is repulsive from $\mathrm{HF}$ and from the other functionals, becomes attractive when PW91 is used. This is due to the Pauli repulsion contribution because the electrostatic term remains close to the HF value. The PW91 value of the interaction energy for the benzene- $\mathrm{N}_{2}$ system is smaller than that reported previously ${ }^{20}$ for this system, but this is most probably due to the basis set. The results concerning the water dimer show that the $6-31++\mathrm{G}(\mathrm{d}, \mathrm{p})$ basis gives a larger interaction energy than the aug-cc-pVTZ one, and the basis used by Wesolowski et al. ${ }^{20}$ is comparable to 6-31G $(d, p)$. The tabulated values also show that $E_{\mathrm{pol}}+E_{\mathrm{ct}}$ is responsible, for all functionals but PW91, of the largest increase of the stabilizing energy contribution when going from HF to DFT. Moreover, all functionals give, as in the case of the water dimer, a much larger increase of the charge transfer than that of the polarization term. It gives an extra stabilization energy varying between 0.1 and $0.2 \mathrm{kcal} / \mathrm{mol}$ with respect to $\mathrm{HF}$. As for the benzene-methane complex, the BSSE at the DFT level offsets the increase of the charge-transfer term which occurs going from HF to DFT.

As for the other complexes, these results deserve a discussion about the generally observed small $E_{\text {Pauli }}$ values obtained when using PW91. In the SAPT theory, the reference exchange energy is chosen at the HF level and an energy correction is added to the HF initial exchange to obtain the correlated value. As discussed by Stone, ${ }^{1}$ for equilibrium geometries, the "correlated exchange," or correlated Pauli repulsion, appears to be of the same magnitude as the HF one; the repulsive correction varies between 10 and $15 \%$ of the initial HF values. In our calculations, all the functionals, except PW91, give exchange contributions following this assumption. So, the PW91 results appear to be particularly surprising: for example, in the case of the benzene- $\mathrm{N}_{2}$ complex, a decrease of the exchange of $60 \%$ is observed when compared to HF. Another easy way to see the unphysical nature of this result is to consider again an analogy between the CSOV analysis and SAPT. ${ }^{2 \mathrm{c}, 13 \mathrm{c}}$ Here, the stabilization comes from $E_{\mathrm{FC}}$, which is closely related to the first-order SAPT energy. This is in contradiction with the attractive dispersion term, which arises, in the complex, from second-order terms in the perturbation theory (as do polarization and charge transfer; see ref. 1a) and b) for discussion). This kind of results has been recently studied in other articles. ${ }^{36,37}$ It has been shown ${ }^{36}$ that a numerical parallel exists between the PW91 Pauli repulsion energy values and the sum of the SAPT exchange plus dispersion energies. The PW91 stabilization may thus appear to be an artefact. ${ }^{36}$ To conclude on the benzene- $\mathrm{N}_{2}$ complex, we see that, in the case of little polarized interacting fragments of a complex, the available functionals are not able to recover enough energy from the charge transfer/polarization mechanism to give results comparable to post-Hartree-Fock methodologies. This is a well-known weakness of the DFT. ${ }^{6}$
Table 6. Variation of the Interaction Energy Contributions (kcal/mol) Using the DZVP2 Basis Set, in $\left[\mathrm{Cu}^{+}(\operatorname{ImH})_{3}\right]_{2}$ as a Function of the $\mathrm{Cu}-\mathrm{Cu}$ Distance.

\begin{tabular}{|c|c|c|c|c|c|c|}
\hline \multirow{2}{*}{$\begin{array}{l}\begin{array}{l}\mathrm{D}_{\mathrm{Cu}-\mathrm{Cu}} \\
(\AA)\end{array} \\
\text { Method }\end{array}$} & \multicolumn{2}{|c|}{3.6} & \multicolumn{2}{|c|}{4.2} & \multicolumn{2}{|c|}{4.6} \\
\hline & $\begin{array}{c}\text { HF } \\
\text { (MP2) }\end{array}$ & B3LYP & $\begin{array}{c}\mathrm{HF} \\
\text { (MP2) }\end{array}$ & B3LYP & $\begin{array}{c}\text { HF } \\
\text { (MP2) }\end{array}$ & B3LYP \\
\hline$E_{s}$ & 43.2 & 39.4 & 41.2 & 38.2 & 39.6 & 37.0 \\
\hline$E_{\text {Pauli }}$ & 0.4 & 0.3 & 0.0 & 0.1 & 0.0 & 0.2 \\
\hline$E_{\mathrm{FC}}$ & 43.7 & 39.7 & 41.2 & 38.3 & 39.6 & 37.1 \\
\hline$E_{\mathrm{pol}}$ & -2.3 & -2.4 & -1.5 & -1.4 & -1.3 & -1.2 \\
\hline$E_{\mathrm{ct}}$ & -2.7 & -2.8 & -1.7 & -1.6 & -1.0 & -1.0 \\
\hline$E_{\mathrm{pol}}+E_{\mathrm{ct}}$ & -5.0 & -5.2 & -3.2 & -3.0 & -2.3 & -2.2 \\
\hline$\Delta E^{\mathrm{a}}$ & $\begin{array}{c}38.7 \\
(24.5)\end{array}$ & 34.5 & $\begin{array}{c}38.0 \\
(29.5)\end{array}$ & 35.3 & $\begin{array}{c}37.3 \\
(31.2)\end{array}$ & 34.9 \\
\hline
\end{tabular}

${ }^{\text {a }}$ The values in parentheses correspond to the MP2 values.

$$
\left[\mathrm{Cu}-(\mathrm{ImH})_{3}\right]_{2}
$$

In previous studies we had found that the binuclear complex $\left[\mathrm{Cu}^{+}-(\mathrm{ImH})_{3}\right]_{2}$ displayed in Figure $1 \mathrm{~g}$ exhibits a metastable arrangement for a $\mathrm{Cu}-\mathrm{Cu}$ distance of $3.6 \AA$ using the DZVP2 basis set at the B3LYP and MP2 levels. ${ }^{21,22}$ The least stable arrangement is obtained for a distance of $4.2 \AA$. The HF results (Table 6) give the expected regular repulsion decrease when the $\mathrm{Cu}-\mathrm{Cu}$ distance increases over the whole range of distances studied. ${ }^{21}$ To determine the origin of this qualitatively different behavior we undertook HF-CSOV and B3LYP-CSOV computations for three distances encompassing the arrangement corresponding to the local repulsion maximum. The results reported in Table 6 show that, when the $\mathrm{Cu}-\mathrm{Cu}$ distance decreases, the repulsive first-order $E_{\mathrm{FC}}$ undergoes a smaller increase when using DFT. On the contrary $\left(E_{\mathrm{pol}}+E_{\mathrm{TC}}\right)$ undergoes a more rapid increase. Because of these opposite variations the overall repulsive interaction energy is smaller for a $\mathrm{Cu}-\mathrm{Cu}$ distance of $3.6 \AA$ than for $4.2 \AA$ when correlation is taken into account. In this case the charge transfer term has very close values in the two sets of data, and the electrostatic term exhibits the largest difference between the HF and DFT results. On the whole, it can be seen that the existence of the shallow local minimum around $3.6 \AA$ is due to the sum of intra- and intermolecular contributions to the correlation energy stabilization. In this case, the comparison of the DFT with the MP2 energy values deserves two remarks. The first one concerns the distance corresponding to the maximum repulsion, which is larger from the MP2 than from the DFT treatment. The second one concerns the difference between the MP2 and DFT values, which decreases as the $\mathrm{Cu}-\mathrm{Cu}$ distance increases. These two features are somewhat unexpected because DFT is known to underestimate correlation effects as the intermolecular distance increases. Moreover, the apparent numerical difference between the DFT and the MP2 interaction energies is expected to originate from the BSSE, as discussed previously for other compounds. 
Table 7. $\operatorname{ImH}(\mathrm{A}) \ldots \mathrm{Zn}^{++}$(B) Interaction Energy Decomposition (kcal/ mol) Using the DZVP2 Basis Set.

\begin{tabular}{lrrrrr}
\hline Method & HF & PW91 & B3PW91 & BLYP & B3LYP \\
\hline$E_{s}$ & -140.3 & -132.5 & -136.2 & -135.4 & -136.6 \\
$E_{\text {Pauli }}$ & 77.7 & 67.8 & 72.5 & 71.4 & 70.5 \\
$E_{\mathrm{FC}}$ & -62.6 & -64.7 & -63.7 & -64.0 & -66.0 \\
$E_{\mathrm{polB}}$ & -1.9 & -2.3 & -1.8 & -2.1 & -2.2 \\
$E_{\mathrm{polA}}$ & -69.2 & -82.6 & -79.5 & -82.6 & -79.4 \\
$E_{\mathrm{ctB} \rightarrow \mathrm{A}}$ & -2.6 & -3.1 & -3.0 & -3.1 & -3.0 \\
$E_{\mathrm{ctA} \rightarrow \mathrm{B}}$ & -9.8 & -25.6 & -20.3 & -28.0 & -22.4 \\
$E_{\mathrm{pol}}+E_{\mathrm{ct}}$ & -83.6 & -113.6 & -104.6 & -115.9 & -107.0 \\
$\Delta E^{\mathrm{a}}$ & -151.4 & -188.1 & -176.5 & -189.8 & -181.9 \\
& $(-163.5)$ & & & & \\
$\mathrm{BSSE}$ & 3.23 & 3.89 & 3.56 & 4.23 & 3.75 \\
& $(9.5)$ & & & & \\
$\alpha_{\mathrm{A}}\left(\mathrm{au}^{3}\right)$ & 40.90 & 42.36 & 41.72 & 42.61 & 41.80 \\
$\alpha_{\mathrm{B}}\left(\mathrm{au}^{3}\right)$ & 1.30 & 1.82 & 1.63 & 1.91 & 1.70 \\
$\mu_{\mathrm{A}}(\mathrm{D})$ & 4.10 & 3.84 & 3.96 & 3.85 & 3.95 \\
$\mu_{\mathrm{B}}(\mathrm{D})$ & - & - & - & - & - \\
\hline
\end{tabular}

${ }^{\text {a }}$ The values in parentheses correspond to the MP2 values.

\section{$\operatorname{ImH}-\mathrm{Zn}^{++}$}

To close our work, we consider the case of the $\mathrm{ImH}-\mathrm{Zn}^{++}$ complex (Table 7), which will enable us to investigate in more detail the physical insight of our results. All functionals show the general decrease of $E_{s}$. For $E_{\text {Pauli }}$, all functionals give less repulsive energies but still following the observed hierarchy:

$$
\text { BLYP }>\text { B3PW91 > B3LYP }>\text { PW91 }
$$

The large stabilization given by DFT with respect to HF is mainly due to the polarization and charge transfer contributions, which both undergo a very large increase.

Another point is the severe overestimation of the total $\Delta E$ by all functionals when compared to MP2 (up to $26 \mathrm{kcal} / \mathrm{mol}$ ). Moreover, an $\mathrm{AIM}^{38}$ (Atom in Molecules) atomic population analysis (Table 8 ) shows the very similar behavior of the MP2 and DFT

Table 8. Atoms in Molecule (AIM) Populations for the $\mathrm{Zn}^{++}$-Imidazole Complex at the HF, MP2 and PW91 Levels of Calculation.

\begin{tabular}{|c|c|c|c|c|c|c|}
\hline & \multicolumn{3}{|c|}{ Isolated fragments } & \multicolumn{3}{|c|}{ Complex } \\
\hline & MP2 & PW91 & $\mathrm{HF}$ & MP2 & PW91 & $\mathrm{HF}$ \\
\hline $\mathrm{Zn}^{++}$ & 28.00 & 28.00 & 28.00 & 28.43 & 28.53 & 28.36 \\
\hline $\mathrm{H} 1$ & 0.92 & 0.91 & 0.94 & 0.77 & 0.76 & 0.78 \\
\hline $\mathrm{C} 2$ & 5.67 & 5.71 & 5.61 & 5.56 & 5.55 & 5.47 \\
\hline N3 & 8.30 & 8.23 & 8.49 & 8.22 & 8.21 & 8.43 \\
\hline $\mathrm{C} 4$ & 5.04 & 5.10 & 4.81 & 4.98 & 4.98 & 8.43 \\
\hline H5 & 0.53 & 0.55 & 0.51 & 0.43 & 0.43 & 0.41 \\
\hline N6 & 8.12 & 8.09 & 8.34 & 8.31 & 8.26 & 8.53 \\
\hline $\mathrm{H} 7$ & 0.90 & 0.90 & 0.92 & 0.82 & 0.80 & 0.82 \\
\hline $\mathrm{C} 8$ & 5.55 & 5.59 & 5.39 & 5.61 & 5.62 & 5.47 \\
\hline H9 & 0.93 & 0.91 & 0.96 & 0.84 & 0.82 & 0.87 \\
\hline
\end{tabular}

Table 9. Comparison of Distributed and Molecular Polarizabilities $\left(\mathrm{au}^{3}\right)$ of the Imidazole Ligand at the HF, PW91, and MP2 Levels.

\begin{tabular}{lrcr}
\hline Polarizability & HF & DFT PW91 & MP2 \\
\hline N6 (lone pair) & 0.69 & 1.47 & 0.69 \\
Molecular & 41.17 & 42.2 & 41.78 \\
\hline
\end{tabular}

evolutions of the imidazole's atomic populations considered first as isolated, and second, in interaction with the $\mathrm{Zn}^{++}$cation.

These results require a better analysis of the interaction. First, as reported in Table 7, we see that, compared to HF, the molecular polarizability of the imidazole increases at the DFT level in agreement with the trend observed previously for the other complexes. Moreover, the polarization energy increases by more than 10 $\mathrm{kcal} / \mathrm{mol}$ when compared to $\mathrm{HF}$ with no spectacular variation of $E_{s}$. This increase can be explained by the mean of a distributed polarizability analysis at the HF, MP2, and DFT levels on the isolated imidazole using the Garner and Stevens method ${ }^{39}$ (polarizabilities distributed on Boys localized orbitals on the atom, bonds, and lone pairs) as implemented (J. P. Piquemal, in preparation) in HONDO.

The results (Table 9) show a huge increase of the PW91 polarizability of the lone pair on atom N6 (Fig. 2), which is the one implied in the $\mathrm{ImH}-\mathrm{Zn}^{++}$interaction. This increase does not occur on this atom at the MP2 level, the repartition of the polarizability gain being more equally spread over all orbitals.

It is then easy to understand that applying the strong electric field generated by the +2 charge of the $\mathrm{Zn}^{++}$cation will generate a huge polarization gain, which gives an explanation for the increased values of $E_{\mathrm{pol}}(\mathrm{A})$ at the DFT level. This result shows that the correlation is not acting in an isotropic way. In fact, this result (as well as all our results) is a consequence of a more complex mechanism directly related to the intimate machinery of the KohnSham approach.

Applying an electric field to a molecule generates a response depolarizing field counteracting the applied one. It has been shown, however, that the expected response, the one obtained from the exact exchange-correlation potential, is not accounted for by the usual GGA functionals. ${ }^{40,41}$ Consequently, the commonly used

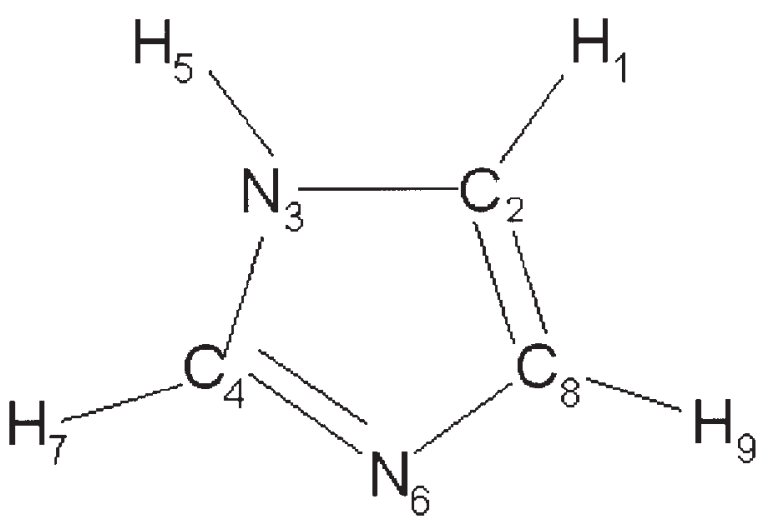

Figure 2. Labeling for the imidazole ( $\mathrm{ImH})$ ligand. 
functionals tend to overestimate polarizabilities. Recent studies have shown that it is possible to calculate good DFT polarizabilities if an additional potential ${ }^{41}$ mimicking the response of the exact exchange-correlation potential is generated. This phenomenon is closely related to the well-known presence of self-interaction in DFT, which arises from the nonproper cancellation between Coulomb and exchange self-interaction, whereas this cancellation is achieved at the HF level. This induces a misrepresentation of the HOMO-LUMO gap, and enhances charge-transfer. Moreover, Zhang et al. ${ }^{42}$ have shown that the charge transfer can be larger in case of fractional populations because of a larger self-interaction error for these kinds of systems. This can explain why the large HF charge transfer in the $\mathrm{ImH}-\mathrm{Zn}^{++}$complex will be considerably enhanced at the DFT level. Following the same idea, another phenomenon can be observed for this strongly interacting complex and concerns the value of $\delta E$. Negligible (inferior to $0.05 \mathrm{kcal} /$ $\mathrm{mol}$ ) for the other investigated complexes, it becomes here important. Starting from $5.2 \mathrm{kcal} / \mathrm{mol}$ at the HF level, $\delta E$ increases at the DFT level to values ranging from 7 to $9 \mathrm{kcal} / \mathrm{mol}$, depending on the functional. This increase is directly related to that of the polarization and is the consequence of the neglect, by the present CSOV approach, ${ }^{12 \mathrm{~b}}$ of the induced dipoles, which are taken into account in the full SCF supermolecule calculation.

Finally, it is important to focus on the response depolarizing field of the exact exchange-correlation potential. It has also been shown that it is intimately related to the Pauli repulsion between closed shells. ${ }^{40}$ The error on polarizabilities can thus be directly linked to the Pauli repulsion value. Our results support this assumption. Indeed, the greatest observed deviations for the polarizabilities from the HF values are obtained for the BLYP and PW91 functionals. The explanation is simple: these two functionals are more subject to self-interaction because they do not contain any exact Hartree-Fock exchange, at variance with B3LYP and B3PW91. This is also in agreement with the fact that BLYP and PW91 give the largest $E_{\mathrm{pol}}$ and $E_{\mathrm{ct}}$ energies.

\section{Concluding Remarks}

In this study, we have shown that several conclusions can be drawn from the comparison of CSOV decompositions relying on $\mathrm{HF}$ and GGA-DFT levels.

First, the results show that the correlation contribution to $E_{\mathrm{FC}}$, which corresponds to intramolecular correlation, can be stabilizing as well as destabilizing. The electrostatic term tends to decrease when correlation is taken into account, while the Pauli repulsion/ exchange term seems very sensitive to the functional used. For example, in the case of the $\left[\mathrm{Cu}^{+}-(\operatorname{ImH})_{3}\right]_{2}$ binuclear complex, our results indicate that the decrease of the electrostatic repulsion between the two positively charged monomers due to intramolecular correlation is important for the existence of a metastable arrangement of this a priori unstable system.

A hierarchy for the value of $E_{\text {Pauli }}$ can be drawn between the functionals: BLYP is the most repulsive followed by B3PW91. B3LYP always remains close to the HF value but exhibits smaller values, while PW91 always provides to weak $E_{\text {Pauli }}$ energies. In general, even if some exceptions have been found $\left(\mathrm{ImH}-\mathrm{Zn}^{2+}\right)$, the HF values can be included in this hierarchy:

$$
\text { BLYP }>\text { B3PW91 }>\text { HF }>\text { B3LYP }>\text { PW91 }
$$

For the systems that we have considered, it appears that PW91 is the functional for which the correlation contribution to the intermolecular binding is the largest: we have discussed the lack of physical meaning of its $E_{\text {Pauli }}$ energies. For the geometry corresponding to the energy minimum of stable complexes (the fragments being frozen to their own equilibrium geometry), our results show that the increase of $E_{\mathrm{pol}}+E_{\mathrm{ct}}$, namely the intermolecular correlation contribution, is mainly due to the increase of the charge transfer term. For some of the functionals and basis sets, it is multiplied by more than a factor 2 in the case of the water dimer or the ImH- $\mathrm{Zn}^{++}$complex. Runenberg et al. ${ }^{9}$ have reported a similar result: for $\left[\mathrm{H}-\mathrm{AuPH}_{3}\right]_{2}$ the so-called "ionic correlation contribution," which is analogous in the LMP2 treatment to the variation of the CSOV charge transfer, becomes very important at short intermolecular distances. For the hydrogen-bonded complexes, the results show that the charge transfer clearly overcomes the lack of dispersion terms because of the presence of self-interaction effects, and can be related to the overlap dispersive effects discussed in the literature. ${ }^{43}$ Nevertheless, for nonpolar systems, the commonly used functionals are unable to generate extra stabilization. More generally, these results point out the fact that DFT replaces true additive dispersive terms by many-body terms such as charge transfer and polarization. This mechanism leads, in some cases, to artifacts. Moreover, it should be kept in mind that the GGA functionals considered here are known to violate some theoretical principles of DFT: ${ }^{37}$ tests on more recent functionals might be useful.

Nevertheless, the Kohn-Sham approach gives very superior results when compared to HF: it appears as the only pragmatic approach available to include dynamical correlation in calculations on large systems.

For the specific purpose of the development of a molecular mechanics systematic parameterized on correlated intermolecular interaction energy decompositions, several points have to be kept in mind. First, the choice of the functional is crucial: our results show that PW91 should be used with caution and that B3LYP may be a reasonable choice, especially due to its $E_{\text {Pauli }}$ behavior which is close to that of HF. The results on imidazole point out the need for a good distribution of the distributed polarizabilities. Finally, we show that any attempt to mimic DFT results through force fields obtained with the usual basis sets must include a charge transfer contribution. Neglecting it can lead to an error that can amount to $25 \mathrm{kcal} / \mathrm{mol}$ of the interaction energy (cf. the ImH- $\mathrm{Zn}^{++}$complex). Such an error cannot be overcome by the sole polarization term which does not have the short-range behavior of the charge transfer term and which does not correspond to the full SAPT induction. Moreover, the usual idea of the nonexistence of a real charge transfer component because of its theoretical disappearance at the Complete-Basis-Set (CBS) limit must be handled with caution. In standard calculations, especially for large molecules, the CBS limit is unreachable and cannot even be reached easily for the water dimer.

Finally, in the very particular cases where the dispersion contribution is the leading component of the total intermolecular interaction energy, it remains possible to include and fit a specific 
long-range dispersion term to be used in molecular mechanics potentials. ${ }^{19,35}$

\section{Acknowledgments}

The computations have been carried out at IDRIS (F. 91403 Orsay, France), CCR (Université Pierre et Marie Curie, F. 75005 Paris, France), CRIHAN (F. 76800 Saint-Etienne-de-Rouvray, France), and NIEHS (North Carolina).

\section{References}

1. (a) Stone, A. J. The Theory of Intermolecular Forces; Oxford University Press: Oxford, 2000; (b) Hayes, I. C.; Stone, A. J. Mol Phys 1984, 53,83 .

2. (a) Jeziorski, B.; Moszynski, R.; Szalewiez, K. Chem Rev 1994, 94, 1887; (b) Moszynski, R.; Cybulski, S. M.; Chalasinki, G. J Chem Phys 1994, 100, 4998; (c) Hess, O.; Caffarel, M.; Huiszoon, C.; Claverie, P. J Chem Phys 1990, 92, 6049

3. Schütz, M.; Rauhut, G.; Werner, H.-J. J Phys Chem A 1998, 102, 5997.

4. Reinhardt, P.; Malrieu, J.-P. J Chem Phys 1998, 109, 7632.

5. Sœbø, S.; Pulay, P. Annu Rev Phys Chem 1993, 44, 213.

6. Langlet, J.; Caillet, J.; Bergès, J.; Reinhardt, P. J Chem Phys 2003, $118,6157$.

7. Reinhardt, P. Chem Phys Lett 2003, 370, 338.

8. Milet, A.; Moszynski, R.; Wormer, P. E. S.; van der Avoird, A. J Phys Chem A 1999, 103, 6811.

9. Runenberg, N.; Schütz, M.; Werner, H.-J. J Chem Phys 1999, 110, 7210.

10. (a) Morokuma, K. J Chem Phys 1971, 55, 1236; (b) Kitaura, K.; Morokuma, K. Int J Quantum Chem 1976, 10, 325; (c) Frey, R.; Davidson, E. R. J Chem Phys 1989, 90, 5555; (d) Cybulski, S.; Scheuner, S. Chem Phys Lett 1990, 166, 57; (e) Gordon, M. S.; Jensen, J. H. In Encyclopedia of Computational Chemistry, von RagueSchleyer, P. J., Ed.; John Wiley and Sons: Chichester, 1998; p. 3198, vol. 5; (f) Morokuma, K.; Pedersen, L. J Chem Phys 1968, 48, 3275 , and references therein.

11. Ziegler, T.; Rauk, A. Theoret Chim Acta 1977, 46, 1

12. (a) Bagus, P. S.; Hermann, K.; Bauschlicher, C. W., Jr. J Chem Phys 1984, 80, 4378; (b) Bagus, P. S.; Illas, F. J Chem Phys 1992, 96, 463; (c) Neyman, K. M.; Ruzankin, S. Ph.; Rösh, N. Chem Phys Lett 1995, 246, 546; (d) Chung, S. C.; Kruger, S.; Ruzankin, S. Ph.; Pacchioni, G.; Rösh, N. Chem Phys Lett 1996, 109, 248; (e) Márquez, A. M.; López, N.; García-Hernández, M.; Illas, F. Surf Sci 1999, 442, 463; (f) Ricca, A.; Bauschlicher, C. W. J Chem Phys 1994, 100, 2900.

13. (a) Stevens, W. J.; Fink, W. Chem Phys Lett 1987, 139, 15; (b) McWeeny, R. Proc R Soc 1959, A253, 242; (c) McWeeny, R.; Ohno, K. A. Proc R Soc 1960, A255, 367; (d) Fink, W. H. J Chem Phys 1972, 57, 1822; (e) Jensen, J. H.; Gordon, M. S. Mol Phys 1996, 89, 1313.

14. Glendening, E. D.; Streitwieser, A. J Chem Phys 1994, 100, 2900.

15. van der Vaart, A.; Merz, K. M., Jr. J Phys Chem A 1999, 103, 3321.

16. Koch, W.; Holthausen, M. C. A Chemist's Guide to Density Functional Theory; Wiley-CH: Weinheim, 2001.

17. Gresh, N. J Chim Phys 1997, 94, 1365.

18. (a) Piquemal, J.-P.; Gresh, N.; Giessner-Prettre, C. J Phys Chem A 2003, 107, 10353; (b) Kairys, V.; Jensen, J. H. Chem Phys Lett 1999,
315, 140; (c) Clementi, E. Computational Aspects of Large Chemical Systems; Springer Verlag: New York, 1980.

19. Adamovic, I.; Freitag, M. A.; Gordon, M. S. J Chem Phys 2003, 118, 6725 .

20. Wesolowski, T. A.; Parisel, O.; Ellinger, Y.; Weber, J. J Phys Chem A 1997, 101, 7818

21. Gresh, N.; Policar, C.; Giessner-Prettre, C. J Phys Chem A 2002, 106, 5660.

22. Piquemal, J.-P.; Maddaluno, J.; Silvi, B.; Giessner-Prettre, C. New J Chem 2003, 27, 909

23. Iikura, H.; Tsuneda, T.; Yanai, T.; Hirao, K. J Chem Phys 2001, 115, 3540.

24. Dupuis, M.; Marquez, A.; Davidson, E. R. "HONDO95.3”, Quantum Chemistry Program Exchange (QCPE); Bloomington, IN: Indiana University.

25. Frisch, M. J.; Trucks, G. W.; Schlegel, H. B.; Scuseria, G. E.; Robb, M. A.; Cheeseman, J. R.; Montgomery, J. A., Jr.; Vreven, T.; Kudin, K. N.; Burant, J. C.; Millam, J. M.; Iyengar, S. S.; Tomasi, J.; Barone, V.; Mennucci, B.; Cossi, M.; Scalmani, G.; Rega, N.; Petersson, G. A.; Nakatsuji, H.; Hada, M.; Ehara, M.; Toyota, K.; Fukuda, R.; Hasegawa, J.; Ishida, M.; Nakajima, T.; Honda, Y.; Kitao, O.; Nakai, H.; Klene, M.; Li, X.; Knox, J. E.; Hratchian, H. P.; Cross, J. B.; Adamo, C.; Jaramillo, J.; Gomperts, R.; Stratmann, R. E.; Yazyev, O.; Austin, A. J.; Cammi, R.; Pomelli, C.; Ochterski, J. W.; Ayala, P. Y.; Morokuma, K.; Voth, G. A.; Salvador, P.; Dannenberg, J. J.; Zakrzewski, V. G.; Dapprich, S.; Daniels, A. D.; Strain, M. C.; Farkas, O.; Malick, D. K.; Rabuck, A. D.; Raghavachari, K.; Foresman, J. B.; Ortiz, J. V.; Cui, Q.; Baboul, A. G.; Clifford, S.; Cioslowski, J.; Stefanov, B. B.; Liu, G.; Liashenko, A.; Piskorz, P.; Komarommi, I.; Martin, R. L.; Fox, D. J.; Keith, T.; Al-Laham, M. A.; Peng, C. Y.; Nanayakkara, A.; Challacombe, M.; Gill, P. M. W.; Johnson, B.; Chen, W.; Wong, M. W.; Gonzalez, C.; Pople, J. A. Gaussian 03, Revision A1; Gaussian, Inc.: Pittsburgh, PA, 2003.

26. Dunning, T. H., Jr. J Chem Phys 1989, 90, 1007.

27. Krishnan, R.; Binkley, J. S.; Seeger, R.; Pople, J. A. J Chem Phys 1980, 72, 650 .

28. Clark, T.; Chandrasekhar, J.; Schleyer, P. v. R. J Comp Chem 1983, 4, 294.

29. Hehre, W. J.; Ditchfield, R.; Pople, J. A. J Chem Phys 1972, 56, 2257.

30. Godbout, N.; Salahub, D. R.; Anselm, J.; Wimmer, E. Can J Chem 1992, 70, 560.

31. Gresh, N.; Parisel, O.; Giessner-Prettre, C. J Mol Struct (Theochem) 1999, 458, 27.

32. van Duijeneveld-van de Rijt, J. G. C. M.; Mooij, W. T. M.; van Duijeneveld, F. B. Phys Chem Chem Phys 2003, 5, 11169.

33. (a) Fonseca Guerra, C.; Bickelhaupt, F. M.; Snijders, J. G.; Baerends, E. J. J Am Chem Soc 2000, 122, 4117; (b) Fonseca Guerra, C.; Bickelhaupt, F. M.; Snijders, J. G.; Baerends, E. J. Chem Eur J 1999, 5, 3581.

34. Bickelhaupt, F. M.; Nibbering, N. M. M.; van Wezenbeek, E. M.; Baerends, E. J. J Phys Chem 1992, 96, 4864

35. Gresh, N. J Phys Chem A 1997, 101, 8680.

36. Langlet, J.; Bergès, J.; Reinhardt, P. Chem Phys Lett 2004, 396, 10.

37. Wesolowski, T. A. J Chem Phys 2000, 113, 1666.

38. Bader, R. F. W. Atoms in Molecule: A Quantum Theory; Clarendon Press: Oxford, 1994.

39. Garmer, D. R.; Stevens, W. J. J Phys Chem 1989, 93, 8263.

40. Gritsenko, O. V.; van Gisbergen, S. J. A.; Schipper, R. T.; Baerends, E. J. Phys Rev A 2000, 62, 12507.

41. Mori-Sanchez, P.; Wu, Q.; Yang, W. J Chem Phys 2003, 119, 11001.

42. Zhang, Y.; Yang, W. J Chem Phys 1998, 109, 2604.

43. Kristyán, S.; Pulay, P. Chem Phys Lett 1994, 229, 175. 